\title{
CURSO DE ACTUALIZACIÓN EN ENFERMERÍA EN UN HOSPITAL ESCUELA: LECTURA DE LA INTEGRACIÓN ENSEÑANZA-SERVICIO
}

\section{NURSING COURSE UPDATE IN HOSPITAL - SCHOOL. SUMMARY OF THE SERVICE-LEARNING INTEGRATION}

\author{
Daniel Planelles Gil ${ }^{*}$ \\ ElizABETH BERnARDino** \\ Gisele KNop Aued ${ }^{* * *}$ \\ LILIANA MÜLLER LAROCCA ${ }^{* * * *}$
}

\begin{abstract}
RESUMEN
Investigación exploratoria-descriptiva, de abordaje cualitativo con 9 docentes-enfermeros y 17 enfermeros asistenciales que dictaron clases en un curso de actualización de enfermería el año 2010 a los trabajadores de un hospital-escuela del sur de Brasil. El objetivo fue evaluar la integración enseñanza-servicio del curso, a través de la percepción de los instructores (enfermeros docentes y enfermeros asistenciales). Los datos fueron compilados el 2011, a través de la aplicación de un cuestionario con preguntas cerradas y una pregunta abierta: ¿Cómo evalúa usted el curso en relación a la integración escuela-servicio? Para el análisis de los datos se utilizó la técnica de análisis de contenido. Los resultados fueron organizados en dos grupos: el perfil de los participantes (charlistas del curso), lo cual englobó datos sociodemográficos y el segundo grupo acerca de los beneficios y la evaluación del curso en relación a la integración enseñanza-servicio en la visión de esos charlistas. Se considera que el curso promovió de alguna forma la integración enseñanza-servicio, aunque hayan divergencias que necesitan ser discutidas.
\end{abstract}

Palabras clave: Educación en enfermería, integración docente-asistencial, hospitales-escuela.

\begin{abstract}
An exploratory and descriptive investigation, with a a qualitative approach, undertaken with the help of 9 nurseteachers and 17 nurses that taught classes in a course of nursing in 2010 for workers in a nursing school in the South of Brazil. The objective was to evaluate the service and teaching integration, seen through the perspective of the intructors (nurse-teachers and nurses). The data was compiled in 2011, through the participation of a questionnaire that consisted of closed-ended questions and an open question: How do you evaluate the course about the integration between nurses-teachers and nurses? For the data analysis, the technique of content analysis was used. The results were organized into two groups: the profile of the group (instructors of the course), which included social and demographic information; and the second group, had to do with the benefits, and the evaluation of the course in relation to the integration of the teaching and service in the
\end{abstract}

\footnotetext{
*Estudiante de graduación en Enfermería, Universidade Federal do Paraná (UFPR), Curitiba, Brasil. Email: danielplanelle@ gmail.com

${ }_{* *}^{*}$ Doctora, profesora y coordinadora del curso de Enfermería, UFPR, Curitiba, Brasil. Email: elizaber@ufpr.br

${ }^{* * *}$ Mestranda del programa de posgrado en Enfermería, UFPR, Curitiba, Brasil. Email: giseleknop8@bol.com.br

${ }^{* * * *}$ Doctora, profesora del curso de Enfermería, UFPR, Curitiba, Brasil. Email: larocca_m@terra.com.br

Instituciones a las que debe darse crédito: UFPR, Conselho Nacional de Desenvolvimento Científico e Tecnológico (CNPq), Hospital de Clínicas-UFPR (HC-UFPR).
} 
vision of the instructors. It is taken into consideration that this course promoted the integration of service and teaching, but there are still divergences that need to be discussed.

Key words: Education in nursing, teaching care integration, hospitals, teaching.

Fecha recepción: 22/02/13. Fecha aceptación: 09/06/14.

\section{INTRODUCCIÓN}

La integración escuela-servicio puede ser entendida como un trabajo que involucra profesores, alumnos y profesionales de determinada área de actuación (1), sin embargo esa integración va más allá de la que sucede durante la prestación de servicio o en las clases prácticas.

Se discute el tema desde la década del 50, cuando el término Integración Docente-Asistencial (IDA) fue empleado para definir la actuación conjunta de docente y estudiantes en el sector de salud de una Universidad (2). En Brasil, la expresión IDA empezó a ser utilizada a mediados de 1973, cuando el Ministerio del Trabajo y de la Previsión Social propuso que hubiese alguna forma de comunicación entre la coordinación de la asistencia médica del Instituto Nacional de Previsión Social y el sistema de formación de personal. En este mismo momento fueron desarrollados proyectos con acciones entre las instituciones de enseñanza y los servicios, en el área de la salud comunitaria, subsidiados por la Fundación Kellog (2).

En 1981 algunas instituciones universitarias adhirieron al programa IDA, instituido por el Ministerio de la Educación y Cultura, lo cual definió como la junción de esfuerzos en un proceso de creciente articulación entre el centro de enseñanza y el servicio de salud, ajustados a las reales necesidades de la población, la creación de conocimiento y la formación de personal necesarios en un determinado contexto de la práctica profesional y de la educación (2).
A partir de la implantación del Sistema Único de Salud (SUS) en 1990, pero más concretamente en 2003, cuando la Secretaría de Gestión de Trabajo y de la Educación en Salud (SGTES), agregada a la Política Nacional de Educación Permanente y Salud, determinó algunas directrices para que se cumplieran los planes de educación permanente de los profesionales del SUS, destacándose la orientación que abarca la relación del trabajo y las prácticas de formación y de desarrollo profesional con la educación permanente. Esa propuesta sigue la misma línea a los principios y directrices del SUS: atención integral a la salud y creación de la cadena de cuidado progresivo a la salud, la cual propone la horizontalidad del trabajo en forma de red, con la atención primaria, ambulatoria y hospitalaria con la finalidad de una asistencia humanizada y responsable a la salud de la comunidad (3).

Actualmente los conceptos de educación permanente, educación continua y educación en servicio aún siguen en discusión. La educación permanente es la propuesta más actual, toda vez que consiste en incorporar la educación y el aprendizaje, dentro de un contexto real, a la rutina diaria de las organizaciones, prácticas sociales y laborales. Entonces la educación continua es considerada un recurso tradicional, la cual se caracteriza por la continuidad del modelo escolar o académico, enfocada en la actualización del conocimiento y está basada en técnicas de transmisión (4). Por último, la educación en servicio tiene como objetivo desarrollar al profesional según los intereses de la institución en que trabaja, o sea, el entrenamiento 
está más direccionado a la técnica que al conocimiento teórico (5).

Las universidades federales y sus hospitales han estado invirtiendo tanto en la educación por medio de cursos de actualización como en la integración entre escuela y servicio. Esta inversión incluye el pago a los profesionales, infraestructura y beneficios salariales que son incorporados después de la capacitación. Se espera, con estos cursos, que los servidores públicos tengan un aumento de sueldo pero que también se actualicen en relación a los usuarios del sistema de salud.

Los cursos de educación continua del presente artículo, dentro de una Política de Educación Permanente, fueron realizados en varias versiones bajo coordinación de un curso de enfermería de una universidad pública en el sur de Brasil y destinados a los servidores de un hospital-escuela entre los años 2008 y 2011. La carga horaria de cada curso era de 150 h para técnicos y auxiliares de enfermería y 180 h para enfermeros. Para la evaluación de los cursos se realizaron tres investigaciones: la primera evaluó el contenido del curso, la segunda el impacto de éstos en unidades de internación y esta investigación se cierra con la tercera etapa de evaluación que tiene como objetivo evaluar la integración enseñanza-servicio del curso de actualización versión 2010, en la percepción de sus instructores (enfermeros-docentes y los enfermeros asistenciales que trabajan en el hospital).

\section{MATERIAL Y MÉTODO}

Se trata de una investigación exploratoria descriptiva, de abordaje cualitativo. La investigación descriptiva tiene como objetivo describir las características de un determinado fenómeno o población. La finalidad de una investigación exploratoria es aclarar y transformar conceptos, ideas y proporciona una visión general en determinada situación. Normalmente las investigaciones descripti- vas exploratorias, en su mayoría, son utilizadas por investigadores preocupados con la actuación práctica y además son requeridas por organizaciones, como las instituciones educacionales (6).

El abordaje cualitativo se justifica por la intención de comprender el fenómeno desde el punto de vista de los enfermeros asistenciales y enfermeros docentes, y también por discutir los datos objetivos y subjetivos sin que exista la necesidad de utilizar recursos estadísticos.

Los participantes de la investigación fueron los docentes-enfermeros de la Universidade Federal do Paraná (UFPR) y enfermeros asistenciales de un hospital-escuela (Hospital de Clínicas-UFPR) vinculado a esta universidad. Los criterios de inclusión para la investigación fueron: ser enfermero que fuese profesor en el Departamento de Enfermería de la universidad o que fuese enfermero asistencial en el hospital-escuela, que hubiese dado clases en el curso durante el año 2010 y que aceptase participar de la investigación mediante la firma de la Declaración de Consentimiento Informado. Los criterios de exclusión fueron: estar de vacaciones o no estar trabajando en la época de la colecta de datos. Como todos los que estaban dentro de los criterios de inclusión aceptaron participar de la investigación, los sujetos en total fueron 26, siendo 9 enfermeros-docentes, y 17 enfermeros asistenciales.

Los datos fueron colectados durante los meses de marzo y abril de 2011, con la aplicación de un cuestionario con preguntas cerradas y una pregunta abierta. Los entrevistados fueron invitados y abordados personalmente para responder las preguntas en ambiente silencioso y reservado, procurando mantener su privacidad. Las preguntas cerradas caracterizaron a los participantes en cuanto a la edad, sexo, calificación y experiencia en la docencia y en la asistencia. La pregunta abierta se hizo solamente con un cuestionamiento a los participantes: ¿Cómo evalúa usted el curso en relación a la integración es- 
cuela-servicio?

Los cuestionarios respondidos fueron analizados por la técnica de Análisis de Contenido de Bardin (7), por modalidad temática, presentados en forma de cuadros para organizar y categorizar las respuestas. El análisis de contenido temático se fundamenta en la división del texto por unidades, en categorías según reagrupamientos analógicos, y compuesto por tres etapas denominadas preanálisis, explotación del contenido y tratamiento de los resultados. Todas las etapas fueron rigurosamente cumplidas y los resultados fueron consensuales entre los autores para garantizar el rigor del método.

La investigación siguió preceptos de la resolución 196/96 del Consejo Nacional de Salud (CNS) (8) y aprobada con el numero $2418.025 / 2011$.

\section{RESULTADOS}

Para responder al objetivo de la investigación, los resultados fueron organizados en dos grupos: el perfil de los participantes (charlistas del curso) y si hubo un contacto entre ellos antes del curso, y el segundo grupo sobre la evaluación del curso en relación a la integración enseñanza/servicio según la visión de esos charlistas.

Los resultados acerca del perfil de los participantes mostraron que los enfermeros-docentes tenían en promedio 43 años y los enfermeros que trabajan en el hospital un promedio de 42 , siendo en su mayoría de sexo femenino, en ambas categorías (docentes y asistenciales). El tiempo medio de actividad de esos profesionales en sus áreas es de 15 años para los maestros y de 14 años para los enfermeros asistenciales. En cuanto al tema de títulos de posgrado, 4 docentes tenían el máster y 5 doctorado, en relación con la categoría asistencial 10 tenían especialidad y 7 máster. Todos los docentes que respondieron al cuestionario tuvieron experiencia con la práctica asistencial de enfermería en promedio 8 años. Los enfermeros de la práctica asistencial vivenciaron la docencia en escuelas privadas como una segunda actividad, en promedio 8 años.

En cuanto al contacto previo entre los instructores, se verificó que 3 docentes no lo habían tenido (un tercio de los docentes entrevistados). Los demás lo tuvieron durante las prácticas de la graduación, en proyectos de investigación, cursos y también como ex funcionario del hospital. En relación a los enfermeros, 14 habían tenido contacto con los docentes como alumnos de la graduación y posgrado, profesor sustituto o invitado a dictar clases en la graduación. Por lo tanto la experiencia en el ámbito docente y práctica asistencial estuvo presente en los participantes de la investigación.

El grupo de instructores mostró estar calificado, ya que todos los enfermeros asistenciales siguieron los estudios después de haber terminado la graduación, estando 7 en posesión del grado de Maestría. Entre los docentes el título de doctor sobresalió, lo que va en acuerdo con lo que se exige en la academia.

En relación a la evaluación del curso en cuanto a la integración enseñanza/servicio en la perspectiva de sus instructores, fueron construidas cuatro categorías: 1) contribución del curso para la integración entre los docentes del departamento de enfermería de la universidad y los enfermeros asistenciales del hospital-escuela, 2) potencial integrativo del curso de actualización, 3) sugerencias indicadas por los instructores y 4 ) beneficios proporcionados por la integración docente-asistencial.

Cuando los instructores fueron consultados acerca de la contribución del curso para la integración entre el cuerpo docente del departamento de enfermería y los enfermeros asistenciales del hospital-escuela, se obtuvieron respuestas positivas y negativas. Los enfermeros asistenciales que respondieron negativamente acerca de este tema creen que hay una separación visible entre el departa- 
mento de enfermería y el hospital.

En relación al potencial integrativo del curso, todos los enfermeros docentes creen que el Curso de Actualización tiene un potencial integrativo, pero 3 de ellos discuerdan, lo que demuestra la necesidad de presentar otras estrategias para fortalecer el vínculo entre enseñanza-servicio.

Las sugerencias presentadas por los instructores fueron categorizadas y se observan semejanzas entre ellas. Sin embargo, solamente los docentes sugirieron cambios en la metodología del curso, probablemente debido a que esos profesionales tienen mayor familiaridad con el tema y han comprendido la importancia de esos métodos en el proceso de enseñanza-aprendizaje. Los enfermeros asistenciales proponen mejor divulgación, inclusión y amplitud, lo que muestra el reconocimiento de los enfermeros asistenciales en relación a la importancia de los cursos de actualización. Los enfermeros asistenciales evaluaron el curso como enfermeros que actúan en la práctica y no como instructores de curso. Las experiencias que tuvieron como docentes y la vivencia de aquellos que realizaron la Maestría parecen no haber influenciado en sus respuestas. El resumen de las categorías se presenta en la Tabla 1.

Tabla 1. Evaluación del curso en cuanto a la integración enseñanza/servicio en la perspectiva de sus instructores, Curitiba, 2012.

\begin{tabular}{|c|c|c|c|}
\hline Categorías & Subcategorías & Docentes & Enfermeros \\
\hline \multirow{3}{*}{$\begin{array}{l}\text { Contribución } \\
\text { del curso para la } \\
\text { integración entre } \\
\text { los docentes y } \\
\text { los enfermeros } \\
\text { asistenciales }\end{array}$} & \multirow[b]{2}{*}{ Sí } & - Aproximación teórico-práctica; & - Aproximación teórico-práctica; \\
\hline & & $\begin{array}{l}\text { - Conocimiento de las dos } \\
\text { realidades profesionales. }\end{array}$ & $\begin{array}{l}\text { - Conocimiento de las dos } \\
\text { realidades profesionales. }\end{array}$ \\
\hline & No & $\begin{array}{l}\text { - Depende de la postura } \\
\text { adoptada por el profesional, } \\
\text { lo cual contribuirá o no a esa } \\
\text { integración. }\end{array}$ & $\begin{array}{l}\text { - Depende de la postura adoptada } \\
\text { por el profesional y que las } \\
\text { relaciones pueden distanciarse, } \\
\text { cuando están marcadas por el } \\
\text { teoricismo, autoritarismo o por } \\
\text { falta de didáctica. }\end{array}$ \\
\hline \multirow{2}{*}{$\begin{array}{l}\text { Potencial } \\
\text { integrativo } \\
\text { del curso de } \\
\text { actualización }\end{array}$} & Sí & $\begin{array}{l}\text { - Aproximación teórico-práctica; } \\
\text { - Conocimiento de las dos } \\
\text { realidades profesionales. }\end{array}$ & $\begin{array}{l}\text { - } 14 \text { afirmaron la aproximación } \\
\text { teórico-práctica y conocimiento de } \\
\text { las dos realidades profesionales. }\end{array}$ \\
\hline & No & 0 & 3 \\
\hline $\begin{array}{l}\text { Sugerencias } \\
\text { indicadas por los } \\
\text { instructores del } \\
\text { curso }\end{array}$ & - & $\begin{array}{l}\text { - Sugirieron cambios en la } \\
\text { metodología del curso. }\end{array}$ & $\begin{array}{l}\text { - Mejor divulgación, inclusión y } \\
\text { amplitud del curso. }\end{array}$ \\
\hline
\end{tabular}

Acerca de los beneficios proporcionados por la integración docente-asistencial referente al curso de actualización, los datos fueron agrupados por semejanza, emergiendo cuatro categorías: beneficios relacionados a la profesión, a la práctica, al crecimiento profesional y a oportunidades, que en general son similares según su ambiente (Tabla 2 ). 
Tabla 2. Beneficios de la integración docente-asistencial, Curitiba, 2012.

\begin{tabular}{|c|c|c|}
\hline Beneficios & Docentes & Enfermeros \\
\hline $\begin{array}{l}\text { Relacionados a la } \\
\text { profesión (visibilidad) }\end{array}$ & $\begin{array}{l}\text { - Reconocimiento profesional y } \\
\text { fortalecimiento de la profesión de } \\
\text { enfermería; } \\
\text { - Valoración de los profesores frente a } \\
\text { los funcionarios del hospital. }\end{array}$ & $\begin{array}{l}\text { - Crecimiento de la profesión de } \\
\text { enfermería; } \\
\text { - Valoración de los enfermeros } \\
\text { asistenciales frente a los docentes. }\end{array}$ \\
\hline $\begin{array}{l}\text { Relacionados a la } \\
\text { práctica }\end{array}$ & $\begin{array}{l}\text { - Actualización de los docentes y } \\
\text { profesionales; } \\
\text { - Mejorar la calidad del cuidado y } \\
\text { asistencia. }\end{array}$ & $\begin{array}{l}\text { - Actualización del profesional; } \\
\text { - La mejora de la práctica asistencial. }\end{array}$ \\
\hline $\begin{array}{l}\text { Crecimiento } \\
\text { profesional (saberes) }\end{array}$ & $\begin{array}{l}\text { - Base teórica a los asistenciales para } \\
\text { que den sentido a la relación teórico- } \\
\text { práctica. }\end{array}$ & $\begin{array}{l}\text { - Divulgación de trabajos de los } \\
\text { docentes y enfermeros asistenciales. }\end{array}$ \\
\hline Oportunidades & $\begin{array}{l}\text { - Envolvimiento y colaboración del } \\
\text { sector administrativo; } \\
\text { - Realizar proyectos; } \\
\text { - Convergencia de cultura, creación de } \\
\text { espacios para discusión, más diálogo. }\end{array}$ & $\begin{array}{l}\text { - Cambio de experiencias docente- } \\
\text { asistencial; } \\
\text { - Participación y desarrollo de } \\
\text { investigación y trabajos; } \\
\text { - Docente trabajando con los } \\
\text { enfermeros del hospital; } \\
\text { - Integración con los alumnos de } \\
\text { prácticas. }\end{array}$ \\
\hline
\end{tabular}

Los resultados acerca de los beneficios de la integración docente-asistencial van al encuentro de la visibilidad, una vez que es de interés común el desarrollo de la profesión de enfermería.

Se confirma que los dos grupos se sienten desvalorizados y apuestan al curso como un medio de obtener esta valorización, desviándose del objetivo mayor, la integración de los grupos para la potencialización de la profesión.

Los beneficios referentes a la práctica revelaron que el curso promovió la actualización de los docentes y de los profesionales, ya que la gran mayoría de los cursos de este tipo proporciona la actualización de los involucrados, posibilitando el aprendizaje al compartir experiencias y saberes. Además, las discusiones teórico-prácticas que surgen en estos espacios son enriquecedoras, pudiendo mejorar o transformar la conducta de docentes y enfermeros asistenciales.

\section{DISCUSIÓN Y CONCLUSIÓN}

Lo resultados mostraron enfermeros maduros, con experiencia, en la misma etapa media de edad y en su mayoría del sexo femenino. La presencia del sexo femenino como predominante en el cuadro de funcionarios entre los docentes y enfermeros asistenciales puede ser comprendida por los aspectos sociohistóricos provenientes de las instituciones de las órdenes religiosas, de la figura mujer-madre, la acción caritativa, entre otras cualidades presentadas en su historicidad. También se puede agregar la influencia de Florence Nightingale, representada como la creadora de la enfermería, al instituir la esencia femenina como factor determinante en la práctica de enfermería (9).

La Ley 9394/96, de las Directrices y Bases de la Educación Nacional, establece que por lo menos un tercio del cuerpo docente tenga 
el título de Mestre y Doctor (10), correspondiendo a la formación académica de los enfermeros docentes que fueron entrevistados. Sin embargo, no existe una exigencia de títulos de maestría o doctorado para el ejercicio de enfermería en los hospitales-escuela, pero sí hay un incentivo del gobierno para la calificación. Se advirtió que los enfermeros que trabajan en el hospital poseen el título por ese estímulo financiero a los profesionales que tengan un curso de posgraduación. Por eso, tal incentivo es responsable por la transformación del perfil de los enfermeros asistenciales, aunque no se conozca el impacto de ese cambio en la práctica asistencial.

La experiencia en el ámbito docente y práctica asistencial estuvo presente en los participantes de la investigación, lo que puede contribuir para la integración entre ambas categorías, en especial porque todos ya vivenciaron las dos posiciones, conociendo las posibilidades y dificultades de cada área. Se observó en este caso que los enfermeros asistenciales ejercieron la docencia en escuelas privadas de enfermería, lo que va al encuentro de la creciente expansión de esas escuelas en Brasil y la necesidad emergente de profesores, ya que para las organizaciones académicas y asistenciales de enfermería el trabajo conjunto del enfermero docente y el enfermero de la práctica clínica representa una estrategia de crecimiento, valorización profesional y de la profesión (11).

Los datos del censo educacional brasileño de 2009 revelan que, de los 3.381 cursos superiores del área de Salud y Bienestar Social, 752 eran de enfermería, siendo que 604 pertenecen a la red privada y que están divididos en 489 instituciones de enseñanza superior particular y 115 en instituciones de enseñanza superior comunitarias, confesionales o filantrópicas (12).

El contacto previo entre la mayoría de los participantes de la investigación se presentó como un factor positivo para la integración entre ellos. Ocurre que cuando los participantes no se conocen, el proceso de inte- gración se vuelve más lento y para que eso no suceda, deben de ser superadas barreras como la timidez y el desconocimiento del área de actuación de cada profesional.

Al principio, la presencia de docentes y alumnos en un hospital-escuela puede ser comprendida como participación en la formación de la práctica hospitalaria, pero frecuentemente el maestro puede ser visto como el que detiene el conocimiento y el poder, dificultando la relación con los enfermeros del hospital. El Curso de Actualización, además de proveer la actualización de los profesionales de la salud, propició de alguna manera la integración, pero algunas diferencias en la enseñanza de la práctica profesional han generado discusiones acerca de la integración escuela-servicio.

Son muchos los conflictos que emergen de esta integración. Por un lado están los trabajadores que se quejan de la desconsideración de las instituciones de enseñanza con los funcionarios del hospital, por otro lado los profesionales del hospital se sienten recriminados por solamente contribuir en la supervisión del alumno, realizada de forma asistemática. No es diferente a lo que pasa con la organización hospitalaria y la institución de enseñanza, en cuanto que la primera es criticada por tener el enfoque en la productividad de sus procedimientos técnico-operacionales y la segunda criticada por centralizar su producción en el conocimiento teórico (1). Se especula que esas dificultades derivan de la diferencia entre los retos metodológicos de la academia y del servicio hospitalario, como también la falta de cultura de determinados profesionales del hospital y de la escuela de enfermería al realizar actividades multiprofesionales, planeadas dentro de una visión interdisciplinar (13).

En un estudio de caso en el cual se describe la integración docente-asistencial de la escuela de enfermería de la Universidade Federal do Rio Grande do Sul con el Hospital de Clínicas de Porto Alegre, los autores señalan que el conflicto por el poder y el estatus de- 
muestra la división teórico-práctica del trabajo, resultando en una relación de dominio, resistencia y subordinación (14).

Se observó que la contribución integradora entre los profesores de UFPR y los enfermeros asistenciales del Hospital de Clínicas-UFPR converge con el estudio de caso cuando se menciona esa dicotomía entre servicio-enseñanza, pero se distancia cuando el resultado de esta investigación apunta a cómo puede ser positiva la relación docente-asistencial, ya que para ambas partes favorece el contacto, aproximación, conocimiento y actualización de las dos realidades.

Lo ideal es que haya integración de la docencia con los discentes y con los profesionales de la práctica asistencial, colaborando la integralidad de las acciones. Además, el currículo programático debería interactuar con el servicio, la enseñanza y la comunidad, con la finalidad de problematizar y crear un aprendizaje crítico-reflexivo, transformando nuevos perfiles de trabajo y de profesionales (1).

Con la integración enseñanza-servicio las actuación de los docentes y de los profesionales del hospital puede ser entendida como un proceso solidario de acción-reflexión-acción, de cuestionamiento y experimentación, en lo cual todos los participantes enseñan y aprenden, interviniendo para ayudar y promocionar el aprendizaje y no para imponer o sustituir la comprensión (15).

Además de los cursos de actualización a los profesionales de la institución de enseñanza, pueden ser implementadas innumerables estrategias con la finalidad de integración, como: curso de actualización del docente con la participación de los profesionales de la práctica asistencial, promover eventos juntos con las instituciones de salud, ofrecer material gráfico para las instituciones de salud, entre otros (16).

Profesionales de la salud que vivencian la integración enseñanza-servicio comentan que la creación de las relaciones horizontales y la apuesta en la construcción de lo nuevo, donde los resultados sean compartidos, derivan de los esfuerzos emprendidos. Ese es el mejor camino posible, aunque no esté construido, pero que es reconstruido por cada persona involucrada, en cada proceso negociado (17).

Docentes de un estudio acerca de la integración docente-asistencial apuntan que la integración puede traer beneficios significativos para las instituciones de enseñanza, servicios hospitalarios y docentes. La construcción de este proceso indica una oportunidad de actualización de los docentes, lo que se reflejará en avances de la calidad de la enseñanza (18).

Desde 1968, con la reforma universitaria brasileña, la integración docente-asistencial es considerada factor importante para la calificación de futuros profesionales y la mejora de la calidad de asistencia en enfermería (18), toda vez que se cree que la potencialización de conocimientos generada por la integración docente-asistencial es lo que contribuye para elevar el nivel de calidad en la prestación de la asistencia.

Además, la inserción de los procesos de enseñanza dentro de las instituciones de salud son esenciales para lograr dos transformaciones primordiales del SUS: modificar el proceso de trabajo al mismo tiempo que se cambia el paradigma flexneriano de la formación académica en el área de la salud (19).

En cuanto a los saberes, se constata que los docentes se sienten superiores en relación a los enfermeros asistenciales, toda vez que tienen la intención de basarlos teóricamente; todavía se debe considerar que los enfermeros asistenciales, que pertenecen al grupo de instructores, también poseen base teórica, la cual en la mayoría de las ocaciones es aplicada, testada y validada en la práctica. A diferencia de los docentes, los enfermeros asistenciales afirmaron que el curso favoreció la difusión de los trabajos de docentes y enfermeros, lo que es importante para el conocimiento y comprensión del trabajo de cada uno y oportunamente crear ligazones que fa- 
vorezcan el desarrollo teórico-práctico.

Se observó que la integración puede resultar también en mayor involucramiento del cuerpo docente con el servicio, que podría ser un facilitador en la realización de nuevos proyectos, investigaciones, trabajos e intercambios de experiencias. Surgió como oportunidad el entendimiento entre los dos grupos en el ejercicio de sus actividades, en espacios para discusiones e integración de la tríada docencia-discencia-asistencia.

En otro estudio acerca de la integración docente-asistencial se considera el proceso extremadamente relevante, así como un desafío que debe ser superado, tanto por los enfermeros docentes como por los enfermeros asistenciales, y agregan que esta integración puede aproximar la relación entre los liderazgos de las instituciones de enseñanza y de los servicios con la intención de mejorar el proceso de enseñanza-aprendizaje de enfermería. Además la integración puede favorecer el cambio de conocimientos entre docentes y enfermeros y propiciar una mejor formación de los estudiantes de enfermería (20).

Es necesaria la discusión entre docentes y enfermeros asistenciales acerca de los retos, las expectativas y de la operacionalización de la IDA, para que la propuesta se acerque más a la realidad (18). Por medio de la discusión es posible extraer los temas que necesitan ser explorados, seleccionar el público que necesita ser involucrado y sobre todo identificar docentes y enfermeros asistenciales que puedan trabajar juntos.

En la perspectiva de los instructores, el curso de actualización promovió la integración enseñanza-servicio, pero aún hay divergencias que necesitan ser discutidas. Varios factores relacionados al perfil de los participantes del estudio podrían prever una mejor integración, como por ejemplo, la experiencia tanto de la docencia como de la práctica asistencial y la buena calificación de los dos grupos.

El acceso a la calificación de los enfermeros de la práctica, por medio del incentivo del gobierno federal, confiere el reconocimiento que le es asignado. Además, el acceso no califica sólo en el cuidado, sino también proporciona mejor habilidad con proyectos de investigación e involucramiento en actividades de la academia, lo que aumenta la autoestima y seguridad de esos profesionales. Por lo tanto, enfermeros asistenciales y docentes se encuentran en el mismo nivel, no habiendo motivos para que no se cooperen para la integración.

Las dificultades en el proceso de integración docente-asistencial aún están presentes, entre ellas se verifica la polarización de posiciones, comprometiendo el avance en el proceso de integración. Para alcanzar la integración, la valoración debe de ser conquistada por el grupo de docentes y enfermeros asistenciales, en la ausencia de la individualidad.

Se percibe que, siendo instructores del curso de actualización, los enfermeros asistenciales hicieron la evaluación según su posición ocupada en el contexto de las organizaciones, demostrando la dificultad de sentirse educador y consecuentemente mejorar la integración enseñanza-asistencia.

Es fundamental ampliar espacios de diálogo entre los dos grupos para disminuir las divergencias aún presentes, toda vez que los conocimientos producidos por las dos dimensiones son complementarios y esenciales para la prestación de cuidados en salud y para mejorar el proceso de formación de futuros profesionales.

\section{REFERENCIAS}

1. Albuquerque VS, Gomes AP, Rezende CHA, Sampaio MX, Dias OV, Lugarinho RM. A integração ensino-serviço no contexto dos processos de mudança na formação superior dos profissionais da saúde. Rev bras educ med. 2008; 32(3): 356-362.

2. Ojeda BS, Santos BRL, Eidt OR. A inte- 
gração docente asistencial na enfermagem: delineando possibilidades para uma prática contextualizada. Acta Paul Enf. 2004; 17(4): 432-8.

3. Brasil. Ministério da Saúde. Secretaria de Gestão do trabalho e da Educação na Saúde e Departamento de Gestão da Educação em Saúde. Política Nacional de Educação Permanente em Saúde [Internet]. Brasília: Ministério da Saúde; 2009 [citado 2 mayo 2011]. Disponível em: http://bvsms.saude.gov.br/bvs/publicacoes/politica_nacional_educacao_permanente_saude.pdf

4. Davini MC. Enfoques, problemas e perspectivas na educação permanente dos recursos humanos de saúde. En: Brasil. Ministério da Saúde. Secretaria de Gestão do Trabalho e da Educação na Saúde. Departamento de Gestão da Educação em Saúde. Política Nacional de Educação Permanente em Saúde. [Internet]. Brasília: Ministério da Saúde; 2009 [citado 2 mayo 2011]. Disponível em: http://bvsms.saude.gov.br/bvs/publicacoes/politica_nacional_educacao_permanente_saude.pdf

5. Farah BF. Educação em serviço, educação continuada, educação permanente em saúde: sinónimos ou diferentes concepções. Rev APS. 2003; 6(2): 123-125.

6. Gil A. C. Métodos e técnicas de pesquisa social. 6a. ed. São Paulo: Atlas; 2008.

7. Bardin L. Análise de conteúdo. 5a ed. Lisboa: Ed. Loyola; 2010.

8. Ministério da Saúde (BR), Conselho Nacional de Saúde, Comissão Nacional de Ética em Pesquisa. Resolução No 196 de 10 de outubro de 1996: diretrizes e normas regulamentadoras de pesquisa envolvendo seres humanos. Brasília: MS; 1996.

9. Lopes MJM, Leal SMC. A feminização persistente na qualificação profissional da enfermagem brasileira. Cad Pagu. 2005; 24: 105-125.

10. Brasil Lei No 9.394 de 20 de dezembro de
1996. Estabelece as diretrizes e bases para a educação nacional [Internet]. Brasília: Presidência da República; 1996 [citado 2 mayo 2011]. Disponível em: http://www. planalto.gov.br

11. Mendes IAC, Trevizan MA, Ferraz CA, Higa EFR. Contribuição das disciplinas da organização de aprendizagem ao processo de parceria docente-assistencial na enfermagem. Rev Lat Am. Enfermagem. 2000; 8(2): 47-52.

12. Leonella VM, Neto MVM, Oliveira MAC. A formação superior de Enfermagem no Brasil: uma visão histórica. Rev Esc Enferm USP. 2011; 45(esp.2): 1774-1779.

13. Pizzinato A, Gustavo AS, Santos BRL, Ojeda BS, Ferreira E, Thiesen FV et al. A integração Ensino-Serviço como Estratégiana FormaçãoProfissional para o SUS. Rev Bras Educ Med. 2012; 36(1): 170177.

14. Olschowsky A, Silva GB. Integração docente-assistencial: umestudo de caso. Rev Esc Enferm USP. 2000; 34(2): 128-137.

15. Reibnitz KS. et al. Rede docente assistencial UFSC/SMS de Florianópolis: reflexos da implantação dos projetos Pró-Saúde I e II. Rev Bras Educ Med. 2012; 36(1): 6875.

16. Caetano JA, Diniz RCM, Soares E. Integração docente-assistencial sob a ótica dos profissionais de saúde. Cogitare Enferm. 2009; 14(4): 638-44.

17. Cavalheiro MTP, Guimarães AL. Formação para o SUS e os desafios da Integração Ensino Serviço. Caderno FNEPAS. 2011; (1): 19-27.

18. Shimizu HE. Percepção de docentes do curso de graduação em enfermagem e obstetrícia de uma universidade pública federal sobre a integração docente assistencial. Rev Lat Am Enfermagem. 1999; 7 (5): 51-57.

19. Pereira PG, Fracolli LA. Articulação Ensino-Serviço e Vigilância da Saúde: a percepção de trabalhadores de saúde de um 
distrito escola.Trab. Educ. Saúde. 2011; 9 (1): 63-75.

20. Beccaria LM, Trevizan MA, Janucci MZ. Integração docente-assistencial entre um curso de enfermagem e um hospital de ensino: concepção do processo sob a ótica de docentes, alunos e enfermeiro. Arq Ciênc Saúde. 2006; 13(3). 